\title{
COMPLETELY INTEGRABLE SYSTEMS WITH A SYMMETRY IN COORDINATES*
}

\author{
TOSHIO OSHIMA ${ }^{\dagger}$
}

\begin{abstract}
We explicitly construct the integrals of completely integrable quantum or classical systems whose potential functions are invariant under the action of a classical Weyl group. Our potential functions and integrals are expressed by the Weierstrass elliptic function.
\end{abstract}

1. Introduction. Many completely integrable quantum or classical dynamical systems have been constructed in connection with root systems (cf. [OP1], [OP2], [In]). Consequently most of them are invariant under the action of the corresponding Weyl groups. Our study is to determine all the completely integrable systems with this invariant property.

Let $W$ be the Weyl group of type $A_{n-1}$ with $n \geq 3$ or of type $B_{n}$ with $n \geq 2$ or of type $D_{n}$ with $n \geq 4$. We identify $W$ with the group of the coordinate transformations

$$
\left(x_{1}, \ldots, x_{n}\right) \mapsto\left(\varepsilon_{1} x_{\sigma(1)}, \ldots, \varepsilon_{n} x_{\sigma(n)}\right)
$$

of $\mathbb{R}^{n}$, where $\sigma$ are the elements of the $n$-th permutation group $\mathfrak{S}_{n}$ and

$$
\begin{cases}\varepsilon_{1}=\cdots=\varepsilon_{n}=1 & \text { if } W \text { is of type } A_{n-1}, \\ \varepsilon_{1}= \pm 1, \cdots, \varepsilon_{n}= \pm 1 & \text { if } W \text { is of type } B_{n}, \\ \varepsilon_{1}= \pm 1, \cdots, \varepsilon_{n}= \pm 1 \text { and } \#\left\{i ; \varepsilon_{i}=-1\right\} \text { is even } & \text { if } W \text { is of type } D_{n} .\end{cases}
$$

We study the Schrödinger operator

$$
P=-\frac{1}{2} \sum_{1 \leq j \leq n} \frac{\partial^{2}}{\partial x_{j}^{2}}+R(x)
$$

on $\mathbb{R}^{n}$ with a $W$-invariant potential function $R(x)$ which has enough $W$-invariant commuting differential operators assuring the complete integrability of $P$. To be precise we assume that there exist $W$-invariant differential operators $P_{1}, \ldots, P_{n}$ with

$$
\left[P_{i}, P_{j}\right]=0 \text { for } 1 \leq i<j \leq n
$$

and

$$
P \in \mathbb{C}\left[P_{1}, \ldots, P_{n}\right]
$$

such that

$$
P_{j}=\sum_{1 \leq i_{1}<\cdots<i_{j} \leq n} \partial_{i_{1}} \cdots \partial_{i_{j}}+R_{j} \text { with ord } R_{j}<j \quad \text { for } 1 \leq j \leq n
$$

or

$$
P_{j}=\sum_{1 \leq i_{1}<\cdots<i_{j} \leq n} \partial_{i_{1}}^{2} \cdots \partial_{i_{j}}^{2}+R_{j} \text { with ord } R_{j}<2 j \quad \text { for } 1 \leq j \leq n
$$

\footnotetext{
* Received November 1, 1998; accepted for publication February 9, 1999.

† Department of Mathematical Sciences, University of Tokyo, Komaba, Tokyo 153-8914, Japan (oshima@ms.u-tokyo.ac.jp).
} 
or

$$
\left\{\begin{array}{l}
P_{n}=\partial_{1} \cdots \partial_{n}+R_{n} \text { with ord } R_{n}<n, \\
P_{j}=\sum_{1 \leq i_{1}<\cdots<i_{j} \leq n} \partial_{i_{1}}^{2} \cdots \partial_{i_{j}}^{2}+R_{j} \text { with ord } R_{j}<2 j \text { for } 1 \leq j<n
\end{array}\right.
$$

if the type of $W$ is $A_{n-1}$ or $B_{n}$ or $D_{n}$, respectively. Here $\mathbb{C}\left[P_{1}, \ldots, P_{n}\right]$ is the commutative algebra generated by $P_{1}, \ldots, P_{n}$, ord $R_{j}$ denote the orders of differential operators $R_{j}$ and for simplicity we put $\partial_{i}=\frac{\partial}{\partial x_{i}}$.

We assume that the coefficients of the differential operators are extended to holomorphic functions on a Zariski open subset of an open connected neighborhood of the origin of the complexification $\mathbb{C}^{n}$ of $\mathbb{R}^{n}$.

The main result of our previous paper [OS] is the following:

If $W$ is of type $A_{n-1}$ with $n \geq 3$, then

$$
R(x)=\sum_{1 \leq i<j \leq n} u\left(x_{i}-x_{j}\right)
$$

with

$$
u(t)=C_{1} \wp(t)+C_{2} .
$$

If $W$ is of type $B_{n}$ with $n \geq 2$, then

$$
R(x)=\sum_{1 \leq i<j \leq n}\left(u\left(x_{i}-x_{j}\right)+u\left(x_{i}+x_{j}\right)\right)+\sum_{1 \leq j \leq n} v\left(x_{j}\right) .
$$

Here if $n \geq 3$, we have

$$
\left\{\begin{array}{l}
u(t)=C_{1} \wp(t)+C_{2} \\
v(t)=\frac{C_{3} \wp(t)^{4}+C_{4} \wp(t)^{3}+C_{5} \wp(t)^{2}+C_{6} \wp(t)+C_{7}}{\wp^{\prime}(t)^{2}}
\end{array}\right.
$$

or

$$
u(t)=C_{1} t^{-2}+C_{2} t^{2}+C_{3} \text { and } v(t)=C_{4} t^{-2}+C_{5} t^{2}+C_{6}
$$

or

$$
u(t)=C_{1} \quad \text { and } \quad v(t) \text { is any even function. }
$$

If $W$ is of type $D_{n}$ with $n \geq 4$, then (1.9) holds with $v=0$ and $u$ is given by (1.10) or (1.11).

Here $C_{1}, C_{2}, \ldots$ are complex numbers and $\wp(t)$ is the Weierstrass elliptic function $\wp\left(t \mid 2 \omega_{1}, 2 \omega_{2}\right)$ with primitive half-periods $\omega_{1}$ and $\omega_{2}$, which are allowed to be infinity.

The purpose of this paper is to construct the operators $P_{1}, \ldots, P_{n}$ mentioned above when $u$ or $(u, v)$ is given by (1.8) or (1.10) for any complex numbers $C_{1}, C_{2}, \ldots$ and for any periods of the elliptic function (cf. Theorem 7.2, 7.3 and 7.5), which was announced in [OOS]. Hence we shall have the complete integrability of the corresponding Schrödinger operator (1.1). We remark that if $W$ is of type $A_{n-1}$, the complete integrability and the operators $P_{1}, \ldots, P_{n}$ are already known (cf. [Ca], [Su], [OP2], [OS], [Et], Theorem 3.2 in this paper).

Taking the "classical limit", we shall also obtain the integrals of the Hamiltonian corresponding to the Schrödinger operator (1.1) because of our simple expression of the operators $P_{1}, \ldots, P_{n}$. 
When $W$ is of type $B_{2}$, our argument in this paper is valid but there exist other potentials which assure the complete integrability. This is caused by a symmetry between $u$ and $v$. We shall treat this case in another paper (cf. [OOS], [OO], [Oc]).

If $u$ or $(u, v)$ is given by $(1.11)$, the operators $P_{1}, \ldots, P_{n}$ do not exist in general and then we need $W$-invariant operators of higher orders (cf. [OP2]), which will be discussed in future.

If $(u, v)$ is given by $(1.12)$, the algebra $\mathbb{C}\left[P_{1} \ldots, P_{n}\right]$ equals the totality of $\mathfrak{S}_{n^{-}}$ invariants of $\mathbb{C}\left[-\frac{1}{2} \partial_{1}^{2}+v\left(x_{1}\right), \ldots,-\frac{1}{2} \partial_{n}^{2}+v\left(x_{n}\right)\right]$.

We note that if $2 \omega_{1}=\sqrt{-1} \pi$ and $\omega_{2}=\infty$, then (1.10) is reduced to

$$
\left\{\begin{array}{l}
u(t)=C_{1}^{\prime} \sinh ^{-2} t+C_{2}^{\prime} \\
v(t)=C_{3}^{\prime} \sinh ^{-2} t+C_{4}^{\prime} \sinh ^{-2} 2 t+C_{5}^{\prime} \sinh ^{2} t+C_{6}^{\prime} \sinh ^{2} 2 t+C_{7}^{\prime}
\end{array}\right.
$$

with complex numbers $C_{1}^{\prime}, \ldots, C_{7}^{\prime}$. The system studied by Heckman-Opdam ([He1], [He2], [HO], [Op1] and [Op2]) corresponds to this trigonometric case with $C_{5}^{\prime}=C_{6}^{\prime}=0$ and they proved its complete integrability. When $C_{5}^{\prime}=C_{6}^{\prime}=0$, an explicit form of $P_{1}, \ldots, P_{n}$ is given by [De].

Moreover if $\omega_{1}=\omega_{2}=\infty$, then (1.10) is reduced to

$$
\left\{\begin{array}{l}
u(t)=C_{1}^{\prime} t^{-2}+C_{2}^{\prime} \\
v(t)=C_{3}^{\prime} t^{-2}+C_{4}^{\prime} t^{2}+C_{5}^{\prime} t^{4}+C_{6}^{\prime} t^{6}+C_{7}^{\prime}
\end{array}\right.
$$

Here we quote a result in [OS] for the operator which commutes with the Schrödinger operator $P$ :

If there exists a nonzero constant $\omega$ such that the $W$-invariant differential operators $P_{1}, \ldots, P_{n}$ are invariant by the parallel translation $x_{1} \mapsto x_{1}+\omega$, then any $W$-invariant differential operator $Q$ that is also invariant by the same parallel translation is contained in $\mathbb{C}\left[P_{1}, \ldots, P_{n}\right]$ if $[P, Q]=0$.

After this paper [Os] was written, [Ch] proved the completely integrability of the Schrödinger operator (1.1) with the elliptic potential function attached to the root system. If the root system is of type $B_{n}$ in our situation, the potential considered in [Ch] corresponds to the case where $v(t)=C_{3} \wp(t)+C_{4}$ or $v(t)=C_{3} \wp(2 t)+C_{4}$ in (1.10). The method is quite interesting but different from this note constructing explicitly all the integrals.

Lastly we give a brief overview of the following sections.

In $\S 2$ preliminary remarks are made and two results employed throughout are established.

In $\S 3$ the two fundamental operators $\Delta$ and $\Delta_{n}$ for $A_{n-1}$ and $D_{n}$ are introduced and their commutativity is proved by the results in $\S 2$. An expansion of $\Delta_{n}$ gives the commuting differential operators for $A_{n-1}$.

In $\S 4$ the Schrödinger operator is allowed to have a term in the potential only depending on the particle position through a given function $v$. A functional differential equation (4.4) is established that will ensure the commutativity of the fundamental operators $P$ and $P_{n}$ for $B_{n}$ given by (4.2).

In $\S 5$, using the lemmas in $\S 2$, we establish solutions of the functional differential equation with the assumption $u=w$ which corresponds to the form (1.9).

In $\S 6$ we look at various rational and trigonometric degenerations of the solutions of the functional differential equation.

In $\S 7$ we bring the results of the previous sections together and establish the commuting differential operators $P_{1}, \ldots, P_{n}$ for $B_{n}$ and $D_{n}$. 


\section{Preliminaries.}

First we introduce some notation used in this paper. For an element $w$ of the permutation group $\mathfrak{S}_{n}$ of the set of indices $\{1, \ldots, n\}$, we define $w(i)=i$ for any $i \in \mathbb{Z}$ satisfying $i<1$ or $i>n$ and we identify $\mathfrak{S}_{n}$ with a subgroup of the group of bijective transformations of $\mathbb{Z}$. Then we have naturally $\mathfrak{S}_{k} \subset \mathfrak{S}_{n}$ if $k<n$.

When we distinguish the Weyl group that we are looking at, we denote it by $W\left(A_{n-1}\right), W\left(B_{n}\right)$ or $W\left(D_{n}\right)$ according to its type. Then $W\left(A_{n-1}\right) \simeq \mathfrak{S}_{n}$ and $W\left(A_{n-1}\right) \subset W\left(D_{n}\right) \subset W\left(B_{n}\right)$. We define a homomorphism $\varepsilon$ of $W\left(B_{n}\right)$ to $\{ \pm 1\}$ by

$$
\varepsilon(w)= \begin{cases}1 & \text { if } w \in W\left(D_{n}\right) \\ -1 & \text { if } w \notin W\left(D_{n}\right)\end{cases}
$$

For the coordinate system $\left(x_{1}, \ldots, x_{n}\right)$ of $\mathbb{R}^{n}$ we put

$$
\partial_{i}=\frac{\partial}{\partial x_{i}}, \partial^{\alpha}=\partial_{1}^{\alpha_{1}} \cdots \partial_{n}^{\alpha_{n}} \quad \text { and } \quad|\alpha|=\alpha_{1}+\cdots+\alpha_{n}
$$

Here $\alpha=\left(\alpha_{1}, \ldots, \alpha_{n}\right)$ with non-negative integers $\alpha_{i}$.

Let $P=\sum p_{\alpha}(x) \partial^{\alpha}$ be a differential operator. Then we put

$$
{ }^{t} P=\sum(-1)^{|\alpha|} \partial^{\alpha} p_{\alpha}(x)
$$

and we say that $P$ is self-adjoint if ${ }^{t} P=P$ and skew self-adjoint if ${ }^{t} P=-P$. For $w \in W$ and a differential operator $P$, we denote by $w(P)$ the differential operator corresponding to $P$ under the coordinate transformation $w$ of $\mathbb{R}^{n}$. In particular we define $P^{-}=w^{-}(P)$ by $w^{-} \in W\left(B_{n}\right)$ with $w^{-}\left(x_{1}, \ldots, x_{n}\right)=\left(-x_{1}, \ldots,-x_{n}\right)$ and we call $P$ has an even parity if $P^{-}=P$ and an odd parity if $P^{-}=-P$. Then we note that

$$
{ }^{t}\left({ }^{t} P\right)=\left(P^{-}\right)^{-}=P, \quad{ }^{t}\left(P^{-}\right)=\left({ }^{t} P\right)^{-}, \quad{ }^{t}(P Q)={ }^{t} Q^{t} P, \quad(P Q)^{-}=P^{-} Q^{-} .
$$

In general the suffix $\{1, \ldots, k\}$ of a function or an operator (eg. $Q_{\{1, \ldots, k\}}$ ) means that it is a function or an operator of the variables $x_{1}, \ldots, x_{k}$ invariant under $w \in \mathfrak{S}_{k}$. And for a function or an operator $Q_{\{1, \ldots, k\}}$ and a subset $I$ of $\{1, \ldots, n\}$, we define $Q_{I}=w\left(Q_{\{1, \ldots, k\}}\right)$ if there exist $w \in W\left(A_{n-1}\right) \simeq \mathfrak{S}_{n}$ with $w(\{1, \ldots, k\})=I$.

Now we review the Weierstrass elliptic function (cf. [WW]), which is

$$
\wp\left(z \mid 2 \omega_{1}, 2 \omega_{2}\right)=\frac{1}{z^{2}}+\sum_{\omega \neq 0}\left(\frac{1}{(z-\omega)^{2}}-\frac{1}{\omega^{2}}\right),
$$

where the sum ranges over all periods $\omega=2 m_{1} \omega_{1}+2 m_{2} \omega_{2}\left(m_{1}, m_{2} \in \mathbb{Z}\right)$ except 0 . We define

$$
\omega_{3}=-\left(\omega_{1}+\omega_{2}\right) \text { and } \omega_{4}=0 .
$$

The Weierstrass elliptic function $\wp(t)$ satisfies the differential equation

$$
\begin{aligned}
\left(\wp^{\prime}\right)^{2} & =4 \wp^{3}-g_{2} \wp-g_{3} \\
& =4\left(\wp-e_{1}\right)\left(\wp-e_{2}\right)\left(\wp-e_{3}\right) .
\end{aligned}
$$


Here

$$
\begin{aligned}
g_{2} & =60 \sum_{\omega \neq 0} \omega^{-4}, g_{3}=140 \sum_{\omega \neq 0} \omega^{-6}, \\
\wp\left(\omega_{\nu}\right) & =e_{\nu} \quad \text { for } \nu=1,2,3, \\
e_{1}+e_{2}+e_{3} & =0, e_{1} e_{2}+e_{2} e_{3}+e_{3} e_{1}=-\frac{g_{2}}{4}, e_{1} e_{2} e_{3}=\frac{g_{3}}{4} .
\end{aligned}
$$

Moreover we have important formulas

$$
\begin{gathered}
\left|\begin{array}{lll}
\wp(x) & \wp^{\prime}(x) & 1 \\
\wp(y) & \wp^{\prime}(y) & 1 \\
\wp(z) & \wp^{\prime}(z) & 1
\end{array}\right|=0 \quad \text { if } x+y+z=0 \\
\wp\left(z+\omega_{i}\right)=e_{i}+\frac{\left(e_{i}-e_{j}\right)\left(e_{i}-e_{k}\right)}{\wp(z)-e_{i}} \text { if }\{i, j, k\}=\{1,2,3\}
\end{gathered}
$$

and

$$
\wp(2 z)=\frac{\left(12 \wp(z)^{2}-g_{2}\right)^{2}}{16 \wp^{\prime}(t)^{2}}-2 \wp(z) .
$$

In this paper the periods are allowed to be infinity and hence $g_{1}$ and $g_{2}$ or $e_{1}$ and $e_{2}$ take any complex numbers. Then the condition

$$
\left(e_{1}-e_{2}\right)\left(e_{2}-e_{3}\right)\left(e_{3}-e_{1}\right) \neq 0
$$

holds if and only if the both periods are finite. On the other hand, if $e_{1}=e_{2}=\frac{1}{3} \lambda^{2}$ and $e_{3}=-\frac{2}{3} \lambda^{2}$ with $\lambda \in \mathbb{C}$, then

$$
\wp\left(z \mid \sqrt{-1} \lambda^{-1} \pi, \infty\right)=\lambda^{2} \sinh ^{-2} \lambda z+\frac{1}{3} \lambda^{2} .
$$

In particular, if $e_{1}=e_{2}=e_{3}=0$, we have

$$
\wp(z \mid \infty, \infty)=z^{-2} .
$$

We note that if $(2.10)$ holds, the function $v(t)$ given by $(1.10)$ is rewritten into

$$
v(t)=C_{5}^{\prime}+\sum_{j=1}^{4} C_{j}^{\prime} \wp\left(t+\omega_{j}\right)
$$

with suitable complex numbers $C_{1}^{\prime}, \ldots, C_{5}^{\prime}$ (cf. (2.5) and (2.8)). Moreover for any complex numbers $C_{1}^{\prime \prime}, C_{2}^{\prime \prime}$ and $C_{3}^{\prime \prime}$, it follows from (2.9) that

$$
v(t)=C_{1}^{\prime \prime} \wp(t)+C_{2}^{\prime \prime} \wp(2 t)+C_{3}^{\prime \prime}
$$

is a special case of (1.10) and the complete integrability of the corresponding Schrödinger operator was a question in [OP2].

Now we prepare

LEMMA 2.1. Let $\tilde{v}_{k}(t), \tilde{u}_{i j}(t)$ and $\tilde{w}_{i j}(t)$ be functions with a single variable for $1 \leq i<j \leq 3$ and $1 \leq k \leq 3$. Put $v_{k}=\tilde{v}_{k}\left(x_{k}\right), v_{k}^{\prime}=\tilde{v}_{k}^{\prime}\left(x_{k}\right), u_{i j}=\tilde{u}_{i j}\left(x_{i}-x_{j}\right)$, $u_{i j}^{\prime}=\tilde{u}_{i j}^{\prime}\left(x_{i}-x_{j}\right), w_{i j}=\tilde{w}_{i j}\left(x_{i}+x_{j}\right)$ and $w_{i j}^{\prime}=\tilde{w}_{i j}^{\prime}\left(x_{i}+x_{j}\right)$ for $1 \leq i<j \leq 3$ and 
$1 \leq k \leq 3$. Then we have

$$
\begin{array}{r}
\partial_{1}\left(\left(u_{12}-w_{12}\right)\left(u_{13}-w_{13}\right)\right)+\partial_{2}\left(\left(u_{12}-w_{12}\right)\left(u_{23}-w_{23}\right)\right)+\partial_{3}\left(\left(u_{13}-w_{13}\right)\left(u_{23}-w_{23}\right)\right) \\
\quad=\left|\begin{array}{ccc}
u_{12} & u_{12}^{\prime} & 1 \\
u_{23} & u_{23}^{\prime} & 1 \\
u_{13} & -u_{13}^{\prime} & 1
\end{array}\right|+\left|\begin{array}{ccc}
u_{12} & u_{12}^{\prime} & 1 \\
w_{13} & -w_{13}^{\prime} & 1 \\
w_{23} & w_{23}^{\prime} & 1
\end{array}\right|+\left|\begin{array}{ccc}
u_{23} & u_{23}^{\prime} & 1 \\
w_{12} & -w_{12}^{\prime} & 1 \\
w_{13} & w_{13}^{\prime} & 1
\end{array}\right|+\left|\begin{array}{ccc}
u_{13} & -u_{13}^{\prime} & 1 \\
w_{23} & -w_{23}^{\prime} & 1 \\
w_{12} & w_{12}^{\prime} & 1
\end{array}\right|,
\end{array}
$$

$$
\begin{array}{r}
\partial_{1}\left(\left(u_{12}+w_{12}\right)\left(u_{13}-w_{13}\right)\right)+\partial_{2}\left(\left(u_{12}+w_{12}\right)\left(u_{23}-w_{23}\right)\right)+\partial_{3}\left(\left(u_{13}+w_{13}\right)\left(u_{23}+w_{23}\right)\right) \\
=\left|\begin{array}{ccc}
u_{12} & u_{12}^{\prime} & 1 \\
u_{23} & u_{23}^{\prime} & 1 \\
u_{13} & -u_{13}^{\prime} & 1
\end{array}\right|+\left|\begin{array}{ccc}
u_{12} & u_{12}^{\prime} & 1 \\
w_{13} & -w_{13}^{\prime} & 1 \\
w_{23} & w_{23}^{\prime} & 1
\end{array}\right|-\left|\begin{array}{ccc}
u_{23} & u_{23}^{\prime} & 1 \\
w_{12} & -w_{12}^{\prime} & 1 \\
w_{13} & w_{13}^{\prime} & 1
\end{array}\right|-\left|\begin{array}{ccc}
u_{13} & -u_{13}^{\prime} & 1 \\
w_{23} & -w_{23}^{\prime} & 1 \\
w_{12} & w_{12}^{\prime} & 1
\end{array}\right|
\end{array}
$$

and

$$
\begin{aligned}
& v_{1}^{\prime}\left(u_{12}-w_{12}\right)+2 v_{1}\left(u_{12}^{\prime}-w_{12}^{\prime}\right)+\partial_{2}\left(\left(v_{1}+v_{2}\right)\left(u_{12}+w_{12}\right)-2 v_{1} v_{2}\right) \\
& =\left|\begin{array}{ccc}
v_{1} & v_{1}^{\prime} & 1 \\
v_{2} & -v_{2}^{\prime} & 1 \\
u_{12} & -u_{12}^{\prime} & 1
\end{array}\right|+\left|\begin{array}{ccc}
v_{1} & -v_{1}^{\prime} & 1 \\
v_{2} & -v_{2}^{\prime} & 1 \\
w_{12} & w_{12}^{\prime} & 1
\end{array}\right| .
\end{aligned}
$$

Proof. If we note that $u_{i j}^{\prime}=\partial_{i} u_{i j}=-\partial_{j} u_{i j}$ and $w_{i j}^{\prime}=\partial_{i} w_{i j}=\partial_{j} w_{i j}$, then equalities (2.15), (2.16) and (2.17) are clear by direct calculations.

In the case when $u(t)=C_{1} \wp(t)+C_{2}$, the function $u(t)$ is even and satisfies

$$
\left|\begin{array}{lll}
u(x) & u^{\prime}(x) & 1 \\
u(y) & u^{\prime}(y) & 1 \\
u(z) & u^{\prime}(z) & 1
\end{array}\right|=0 \quad \text { for } x+y+z=0
$$

which is clear from (2.7). Hence we have

Corollary 2.2. For given even functions $u(t), v(t)$ and $w(t)$, put

$$
\begin{aligned}
\phi_{i j} & =u\left(x_{i}-x_{j}\right)+w\left(x_{i}+x_{j}\right), \\
\psi_{i j} & =u\left(x_{i}-x_{j}\right)-w\left(x_{i}+x_{j}\right), \\
v_{k} & =v\left(x_{k}\right) .
\end{aligned}
$$

Then clearly

$$
\phi_{j i}=\phi_{i j}, \psi_{j i}=\psi_{i j} \quad \text { and } \quad \partial_{i} \phi_{i j}+\partial_{j} \psi_{i j}=0
$$

i) If

$$
u(t)=w(t)=C_{1} \wp(t)+C_{2}
$$

or

$$
u(t)=C_{1} \wp(t)+C_{2} \quad \text { and } \quad w(t)=C_{3},
$$

then

$$
\partial_{i}\left(\psi_{i j} \psi_{i k}\right)+\partial_{j}\left(\psi_{i j} \psi_{j k}\right)+\partial_{k}\left(\psi_{i k} \psi_{j k}\right)=0
$$

and

$$
\partial_{i}\left(\phi_{i j} \psi_{i k}\right)+\partial_{j}\left(\phi_{i j} \psi_{j k}\right)+\partial_{k}\left(\phi_{i k} \phi_{j k}\right)=0 .
$$


ii) If

$$
u(t)=v(t)=w(t)=C_{1} \wp(t)+C_{2}
$$

or

$$
u(t)=w(t)=C_{1} \wp(t)+C_{2} \quad \text { and } \quad v(t)=C_{3}
$$

then

$$
\left(\partial_{i} v_{i}\right) \psi_{i j}+2 v_{i}\left(\partial_{i} \psi_{i j}\right)+\partial_{j}\left(\left(v_{i}+v_{j}\right) \phi_{i j}-2 v_{i} v_{j}\right)=0 .
$$

Here the indices $i, j$ and $k$ are mutually different and $C_{1}, C_{2}$ and $C_{3}$ are any complex numbers.

Remark 2.3. The equation (2.18) for $u$ and its generalization are studied by [BP], [BBy], $[\mathrm{OS}]$ in connection with integrable systems and equations similar to those in Corollary 2.2 are discussed in $[\mathrm{BBu}]$

3. A fundamental integral of type $A_{n-1}$ and $D_{n}$.

In this section we use the notation in Corollary 2.2. Put

$$
\psi_{\{1, \ldots, 2 k\}}=\frac{1}{2^{k} k !} \sum_{w \in \mathfrak{S}_{2 k}} w\left(\psi_{12} \psi_{34} \psi_{56} \cdots \psi_{2 k-1,2 k}\right) .
$$

We sometimes denote by $\psi_{i, j}$ in place of $\psi_{i j}$ to distinguish the suffices.

Define

$$
\Delta=-\frac{1}{2} \sum_{j=1}^{n} \partial_{j}^{2}+\sum_{1 \leq i<j \leq n} \phi_{i j}
$$

and

$$
\Delta_{n}=\sum_{0 \leq \nu \leq\left[\frac{n}{2}\right]} \frac{1}{(2 \nu) !(n-2 \nu) !} \sum_{w \in \mathfrak{S}_{n}} w\left(\psi_{\{1, \ldots, 2 \nu\}} \partial_{2 \nu+1} \cdots \partial_{n}\right) .
$$

Let $\bar{\Delta}$ and $\bar{\Delta}_{n}$ be the functions of $(x, \xi)$ obtained by replacing $\partial_{i}$ by $\xi_{i}$ for $i=1, \ldots, n$ in (3.2) and (3.3), respectively.

The Poisson bracket of functions $f(x, \xi)$ and $g(x, \xi)$ is defined by

$$
\{f, g\}=\sum_{j=1}^{n}\left(\frac{\partial f}{\partial \xi_{j}} \frac{\partial g}{\partial x_{j}}-\frac{\partial g}{\partial \xi_{j}} \frac{\partial f}{\partial x_{j}}\right)
$$

Then we have

Proposition 3.1. Suppose $u$ and $w$ are given by (2.21) or (2.22). Then

$$
\left[\Delta_{n}, \Delta\right]=\left\{\bar{\Delta}_{n}, \bar{\Delta}\right\}=0 \text {. }
$$

Proof. Put $Q=\left[\Delta_{n}, \Delta\right]$ and suppose $Q \neq 0$. Since ${ }^{t} \Delta_{n}=(-1)^{n} \Delta_{n}$ and ${ }^{t} \Delta=\Delta$, ${ }^{t} Q={ }^{t}\left[\Delta_{n}, \Delta\right]=-\left[{ }^{t} \Delta_{n},{ }^{t} \Delta\right]=(-1)^{n+1} Q$. Hence the order of $Q$ equals $n-2 m-1$ with a suitable positive integer $m$. Then by using (2.20) and (2.23), the coefficient of $\partial_{2 m+2} \cdots \partial_{n}$ in the $\mathfrak{S}_{n}$-invariant operator $Q$ equals

$$
\sum_{i=1}^{2 m+1} \psi_{\{1, \ldots, 2 m+1\} \backslash\{i\}} \partial_{i} \sum_{\mu<\nu} \phi_{\mu \nu}+\sum_{j=2 m+2}^{n} \partial_{j} \psi_{\{1, \ldots, 2 m+1, j\}}
$$




$$
\begin{aligned}
& =\sum_{i=1}^{2 m+1} \sum_{\substack{j \neq i \\
1 \leq j \leq n}} \psi_{\{1, \ldots, 2 m+1\} \backslash\{i\}} \partial_{i} \phi_{i j}+\sum_{j=2 m+2}^{n} \sum_{i=1}^{2 m+1} \psi_{\{1, \ldots, 2 m+1\} \backslash\{i\}} \partial_{j} \psi_{i j} \\
& =-\sum_{i=1}^{2 m+1} \sum_{\substack{j \neq i \\
1 \leq j \leq 2 m+1}} \psi_{\{1, \ldots, 2 m+1\} \backslash\{i\}} \partial_{j} \psi_{i j} \\
& =-\sum_{i=1}^{2 m+1} \sum_{\substack{j \neq i \\
1 \leq j \leq 2 m+1}} \sum_{\substack{k \neq i, k \neq j \\
1 \leq k \leq 2 m+1}} \psi_{\{1, \ldots, 2 m+1\} \backslash\{i, j, k\}} \psi_{j k} \partial_{j} \psi_{i j} \\
& =-\sum_{1 \leq i<j<k \leq 2 m+1} \psi_{\{1, \ldots, 2 m+1\} \backslash\{i, j, k\}}\left(\partial_{i}\left(\psi_{i j} \psi_{i k}\right)+\partial_{j}\left(\psi_{i j} \psi_{j k}\right)+\partial_{k}\left(\psi_{i k} \psi_{j k}\right)\right) \\
& =0 \text {, }
\end{aligned}
$$

which contradicts to the fact that the order of $Q$ equals $n-2 m-1$. Thus we have $\left[\Delta_{n}, \Delta\right]=0$ and by the same calculation we have also $\left\{\bar{\Delta}_{n}, \bar{\Delta}\right\}=0$.

The following theorem is known but we repeat it here for the completeness.

Theorem 3.2 (Type $A_{n-1}$. [OP2], [OS, Theorem 5.2 and Remark 5.3]). Put

$$
u(t)=C_{1} \wp(t)+C \quad \text { and } \quad w(t)=0 .
$$

Regard $\Delta_{n}$ as a polynomial function of $C$, denote it by $P_{n}(C)$ and put $P_{n-1}(C)=$ $\left[P_{n}(C), x_{1}+\cdots+x_{n}\right]$. Then

$$
\left[P_{n}(C), P_{n}\left(C^{\prime}\right)\right]=\left[P_{n}(C), P_{n-1}\left(C^{\prime}\right)\right]=\left[P_{n-1}(C), P_{n-1}\left(C^{\prime}\right)\right]=0
$$

for any $C, C^{\prime} \in \mathbb{C}$.

Defining $P_{k}$ by $P_{n}(C)=\sum_{0 \leq \nu \leq\left[\frac{n}{2}\right]} P_{n-2 \nu} C^{\nu}$ and $P_{n-1}(C)=\sum_{0 \leq \nu \leq\left[\frac{n-1}{2}\right]}(2 \nu+$ 1) $P_{n-2 \nu-1} C^{\nu}$, we have

$$
\begin{aligned}
P_{k}= & \sum_{0 \leq j \leq\left[\frac{k}{2}\right]} \frac{C_{1}^{j}}{2^{j} j !(k-2 j) !} \sum_{w \in \mathfrak{S}_{n}} w\left(\wp\left(x_{1}-x_{2}\right) \wp\left(x_{3}-x_{4}\right) \cdots\right. \\
& \left.\cdot \wp\left(x_{2 j-1}-x_{2 j}\right) \partial_{2 j+1} \cdots \partial_{k}\right)
\end{aligned}
$$

and $P_{1}, \ldots, P_{n}$ are the required operators for the Schrödinger operator (1.1) with (1.7) and (1.8) when $W$ is of type $A_{n-1}$.

By replacing $\partial_{i}$ and $[$,$] by \xi_{i}$ and $\{$,$\} , respectively, we have the same claim for$ the corresponding Hamiltonian system.

Proof. Put $Q=\left[P_{n}(C), P_{n}\left(C^{\prime}\right)\right]$ and suppose $Q \neq 0$. Since ${ }^{t} P_{n}(C)=P_{n}(C)^{-}$, we have $-{ }^{t} Q=Q^{-}$. By Jacobi's identity for [, ], we have $[Q, \Delta]=0$, which implies that the coefficients of the terms of highest order in $Q$ are polynomial functions of $x$ (cf. [Be, Lemma 2.5] or [OS, Lemma 3.1 and Lemma 3.5]). Hence if $\omega_{1}$ is finite, the coefficients are constant because of their periodicity. Moreover by the analytic continuation we can conclude that the coefficients are constant even if $\omega_{1}=\infty$. This contradicts to ${ }^{t} Q=Q^{-} \neq 0$. Hence we have $\left[P_{n}(C), P_{n}\left(C^{\prime}\right)\right]=0$.

Note that $\left[P_{n-1}(C), \Delta\right]=-\left[\partial_{1}+\cdots+\partial_{n}, P_{n}(C)\right]+\left[\left[P_{n}(C), \Delta\right], x_{1}+\cdots+x_{n}\right]=$ 0 . Hence the same argument as above shows (3.5) by replacing $\left(P_{n}(C), P_{n}\left(C^{\prime}\right)\right)$ by $\left(P_{n}(C), P_{n-1}\left(C^{\prime}\right)\right)$ or $\left(P_{n-1}(C), P_{n-1}\left(C^{\prime}\right)\right)$.

The remaining part of the theorem is clear from the definition of $P_{n}(C)$. 
4. A functional differential equation. Retain the notation in Corollary 2.2 and the previous section and put

$$
\Delta_{\{1, \ldots, k\}}=\sum_{0 \leq \nu \leq\left[\frac{k}{2}\right]} \frac{1}{(2 \nu) !(k-2 \nu) !} \sum_{w \in \mathfrak{S}_{k}} w\left(\psi_{\{1, \ldots, 2 \nu\}} \partial_{2 \nu+1} \cdots \partial_{k}\right)
$$

for $k=1, \ldots, n$ (cf. (3.3)). Then we have easily

LEMMA 4.1.

$$
\begin{gathered}
{\left[\Delta_{\{1, \ldots, k\}}, x_{k}\right]=\Delta_{\{1, \ldots, k-1\}},} \\
\Delta_{\{1, \ldots, k\}}=\Delta_{\{1, \ldots, k-1\}} \partial_{k}+\sum_{1 \leq \nu \leq k-1} \psi_{\nu k} \Delta_{\{1, \ldots, k-1\} \backslash\{\nu\}} .
\end{gathered}
$$

Let $q_{\{1, \ldots, k\}}$ be suitable symmetric functions of $\left(x_{1}, \ldots, x_{k}\right)$ for $k=1, \ldots, n$ and put $q_{\varnothing}=1$. For even functions $v_{j}=v\left(x_{j}\right)$, we examine the condition such that the operators

$$
\begin{aligned}
P & =-\frac{1}{2} \sum_{j=1}^{n} \partial_{j}^{2}+\sum_{1 \leq i<j \leq n} \phi_{i j}+\sum_{j=1}^{n} v_{j}, \\
P_{n} & =\sum_{k=0}^{n} \frac{1}{k !(n-k) !} \sum_{w \in \mathfrak{S}_{n}} w\left(q_{\{1, \ldots, k\}} \Delta_{\{k+1, \ldots, n\}}^{2}\right)
\end{aligned}
$$

satisfy $\left[P_{n}, P\right]=0$. We denote by $\bar{\Delta}_{\{1, \ldots, k\}}$ and $\bar{P}$ and $\bar{P}_{n}$ the functions of $(x, \xi)$ obtained by replacing $\partial_{i}$ by $\xi_{i}$ in the above definition of the corresponding operators. We introduce symmetric functions $T_{\{1, \ldots, k\}}$ of $\left(x_{1}, \ldots, x_{k}\right)$ such that

$$
q_{\{1, \ldots, k\}}=\sum_{I_{1} \amalg \cdots \amalg I_{\nu}=\{1, \ldots, k\}} T_{I_{1}} \cdots T_{I_{\nu}}
$$

where the sum runs over all different partitions of $\{1, \ldots, k\}$. For example

$$
\begin{aligned}
q_{\varnothing} & =T_{\varnothing}=1, \quad q_{\{1\}}=T_{\{1\}}, \quad q_{\{1,2\}}=T_{\{1\}} T_{\{2\}}+T_{\{1,2\}}, \\
q_{\{1,2,3\}} & =T_{\{1\}} T_{\{2\}} T_{\{3\}}+T_{\{1\}} T_{\{2,3\}}+T_{\{2\}} T_{\{3,1\}}+T_{\{3\}} T_{\{1,2\}}+T_{\{1,2,3\}} .
\end{aligned}
$$

THEOREM 4.2. Retain the above notation. Suppose

$$
\left\{\begin{array}{l}
T_{\{1\}}=-2 v_{1}, \\
\partial_{k} T_{\{1, \ldots, k\}}=\sum_{j=1}^{k-1}\left(2 T_{\{1, \ldots, k-1\}}\left(\partial_{j} \psi_{j k}\right)+\left(\partial_{j} T_{\{1, \ldots, k-1\}}\right) \psi_{j k}\right) \\
\quad \text { for } \quad k=2, \ldots, n .
\end{array}\right.
$$

Then $\left[P_{n}, P\right]=\left\{\bar{P}_{n}, \bar{P}\right\}=0$.

Proof. It follows from Proposition 3.1 that

$$
\begin{aligned}
{\left[P_{n}, P\right] } & =\left[\sum_{k=0}^{n} \frac{1}{k !(n-k) !} \sum_{w \in \mathfrak{S}_{n}} w\left(q_{\{1, \ldots, k\}} \Delta_{\{k+1, \ldots, n\}}^{2}\right), P\right] \\
& =\sum_{k=0}^{n} \frac{1}{k !(n-k) !} \sum_{w \in \mathfrak{S}_{n}} w\left(\left[q_{\{1, \ldots, k\}} \Delta_{\{k+1, \ldots, n\}}^{2},\right.\right.
\end{aligned}
$$




$$
\begin{aligned}
& \left.\left.-\frac{1}{2} \partial_{1}^{2}-\cdots-\frac{1}{2} \partial_{k}^{2}+\sum_{\nu=k+1}^{n}\left(v_{\nu}+\sum_{\mu=1}^{k} \phi_{\mu \nu}\right)\right]\right) \\
= & \sum_{k=0}^{n} \frac{1}{k !(n-k) !} \sum_{w \in \mathfrak{S}_{n}} w\left(q_{\{1, \ldots, k\}}\left[\Delta_{\{k+1, \ldots, n\}}^{2}, \sum_{\nu=k+1}^{n}\left(v_{\nu}+\sum_{\mu=1}^{k} \phi_{\mu \nu}\right)\right]\right. \\
& \left.+\frac{1}{2}\left[\partial_{1}^{2}+\cdots+\partial_{k}^{2}, q_{\{1, \ldots, k\}}\right] \Delta_{\{k+1, \ldots, n\}}^{2}\right) .
\end{aligned}
$$

Hence by Lemma 4.1 and (2.20) we have

$$
\begin{aligned}
\left\{\bar{P}_{n}, \bar{P}\right\}= & \sum_{k=0}^{n-1} \frac{1}{k !(n-k-1) !} \sum_{w \in \mathfrak{S}_{n}} w\left(2 q_{\{1, \ldots, k\}}\left(v_{k+1}^{\prime}+\sum_{j=1}^{k} \partial_{k+1} \phi_{j, k+1}\right)\right. \\
& \left.\cdot \bar{\Delta}_{\{k+2, \ldots, n\}} \bar{\Delta}_{\{k+1, \ldots, n\}}\right) \\
& +\sum_{k=1}^{n} \frac{1}{(k-1) !(n-k) !} \sum_{w \in \mathfrak{S}_{n}} w\left(\left(\partial_{k} q_{\{1, \ldots, k\}}\right) \bar{\Delta}_{\{k+1, \ldots, n\}}^{2} \xi_{k}\right) \\
= & \sum_{k=1}^{n} \frac{1}{(k-1) !(n-k) !} \sum_{w \in \mathfrak{S}_{n}} w\left(2 q_{\{1, \ldots, k-1\}}\left(v_{k}^{\prime}+\sum_{j=1}^{k-1} \partial_{k} \phi_{j k}\right)\right. \\
& \left.\cdot \bar{\Delta}_{\{k+1, \ldots, n\}} \bar{\Delta}_{\{k, \ldots, n\}}\right) \\
& -\sum_{k=1}^{n} \frac{1}{(k-1) !(n-k) !} \sum_{w \in \mathfrak{S}_{n}} w\left(( \partial _ { k } q _ { \{ 1 , \ldots , k \} } ) \left(\bar{\Delta}_{\{k, \ldots, n\}}\right.\right. \\
& \left.\left.+\sum_{j=k+1}^{n} \psi_{k j} \bar{\Delta}_{\{k+1, \ldots, n\} \backslash\{j\}}\right) \bar{\Delta}_{\{k+1, \ldots, n\}}\right) \\
= & \sum_{k=1}^{n} \frac{1}{(k-1) !(n-k) !} \sum_{w \in \mathfrak{S}_{n}} w\left(\left(2 q_{\{1, \ldots, k-1\}} v_{k}^{\prime}\right.\right. \\
& -\sum_{j=1}^{k-1}\left(2 q_{\{1, \ldots, k-1\}} \partial_{j} \psi_{j k}+\left(\partial_{j} q_{\{1, \ldots, k-1\}}\right) \psi_{j k}\right) \\
& \\
&
\end{aligned}
$$

Here the last equality follows from

$$
\begin{aligned}
& \sum_{k=1}^{n} \frac{1}{(k-1) !(n-k) !} \sum_{w \in \mathfrak{S}_{n}} w\left(\left(\partial_{k} q_{\{1, \ldots, k\}}\right) \sum_{j=k+1}^{n} \psi_{k j} \bar{\Delta}_{\{k+1, \ldots, n\} \backslash\{j\}} \bar{\Delta}_{\{k+1, \ldots, n\}}\right) \\
& =\sum_{\ell=1}^{n} \frac{1}{(\ell-1) !(n-\ell) !} \sum_{w \in \mathfrak{S}_{n}} w\left(\sum_{i=1}^{\ell-1}\left(\partial_{i} q_{\{1, \ldots, \ell-1\}}\right) \psi_{i \ell} \bar{\Delta}_{\{\ell+1, \ldots, n\}} \bar{\Delta}_{\{\ell, \ldots, n\}}\right) .
\end{aligned}
$$

Hence if $q_{\{1, \ldots, k\}}$ satisfy

$$
\begin{aligned}
\partial_{k} q_{\{1, \ldots, k\}} & =-2 q_{\{1, \ldots, k-1\}} v_{k}^{\prime} \\
& +\sum_{j=1}^{k-1}\left(2 q_{\{1, \ldots, k-1\}}\left(\partial_{j} \psi_{j k}\right)+\left(\partial_{j} q_{\{1, \ldots, k-1\}}\right) \psi_{j k}\right)
\end{aligned}
$$


for $k=1, \ldots, n$, then $\left\{\bar{P}_{n}, \bar{P}\right\}=0$. Under the assumption of the theorem, the right hand side of (4.5) equals

$$
\begin{aligned}
& \sum_{I_{1} \amalg \cdots \amalg I_{\nu}=\{1, \ldots, k-1\}}\left(T_{I_{1}} \cdots T_{I_{\nu}} \partial_{k} T_{\{k\}}\right. \\
+ & \left.\sum_{j=1}^{k-1}\left(2 T_{I_{1}} \cdots T_{I_{\nu}}\left(\partial_{j} \psi_{j k}\right)+\left(\partial_{j}\left(T_{I_{1}} \cdots T_{I_{\nu}}\right)\right) \psi_{j k}\right)\right) \\
= & \sum_{I_{1} \amalg \cdots \amalg I_{\nu}=\{1, \ldots, k-1\}} \partial_{k}\left(T_{I_{1}} \cdots T_{I_{\nu}} T_{\{k\}}+\sum_{\mu=1}^{\nu} T_{I_{1}} \cdots T_{I_{\mu} \cup\{k\}} \cdots T_{I_{\nu}}\right),
\end{aligned}
$$

which equals the left hand side of $(4.5)$ and hence we have $\left\{\bar{P}_{n}, \bar{P}\right\}=0$.

Thus we have

$$
\begin{gathered}
{\left[P_{n}, P\right]=\sum_{k=0}^{n} \frac{1}{k !(n-k) !} \sum_{w \in \mathfrak{S}_{n}} w\left(q_{\{1, \ldots, k\}} \sum_{\nu=k+1}^{n}\left(\partial_{\nu}^{2} v_{\nu}+\sum_{1 \leq \mu \leq k} \partial_{\nu}^{2} \phi_{\mu \nu}\right)\right.} \\
\left.\cdot \Delta_{\{k+1, \ldots, n\} \backslash\{\nu\}}^{2}+\frac{1}{2} \sum_{\nu=1}^{k}\left(\partial_{\nu}^{2} q_{\{1, \ldots, k\}}\right) \Delta_{\{k+1, \ldots, n\}}^{2}\right)
\end{gathered}
$$

and therefore $\left[P_{n}, P\right]$ is clearly self-adjoint. Since $P$ and $P_{n}$ are self-adjoint, $\left[P_{n}, P\right]$ is skew self-adjoint. Hence we can conclude $\left[P_{n}, P\right]=0$.

REMARK 4.3. Let $T_{\{1, \ldots, k\}}^{1}$ and $T_{\{1, \ldots, k\}}^{2}$ be solutions of $(4.4)$ for $(u, v, w)=$ $\left(f, g_{1}, h\right)$ and $\left(f, g_{2}, h\right)$, respectively. Then $C^{k-1} C^{\prime} T_{\{1, \ldots, k\}}^{1}+C^{k-1} C^{\prime \prime} T_{\{1, \ldots, k\}}^{2}$ are solutions for $(u, v, w)=\left(C f, C^{\prime} g_{1}+C^{\prime \prime} g_{2}, C h\right)$. Here $C, C^{\prime}$ and $C^{\prime \prime}$ are any complex numbers and $k=1, \ldots, n$.

\section{Solutions of the functional differential equation.}

In this section we shall construct elliptic solutions of (4.4) in the case when

$$
u(t)=w(t)=C_{\wp}(t)+C^{\prime}
$$

with $C, C^{\prime} \in \mathbb{C}$ (cf. (2.19)).

Retain the notation in the previous section and assume (5.1).

LEMMA 5.1. Under the notation in Corollary 2.2, the functions

$$
\left\{\begin{array}{l}
\Phi_{0}=1, \\
\Phi_{n}=(-1)^{n} \sum_{w \in \mathfrak{S}_{n}} w\left(\phi_{01} \phi_{12} \cdots \phi_{n-1, n}\right) \quad \text { for } n \geq 1
\end{array}\right.
$$

satisfy

$$
\partial_{n} \Phi_{n}=\partial_{0}\left(\Phi_{n-1} \psi_{0 n}\right)+\sum_{j=1}^{n-1}\left(2 \Phi_{n-1}\left(\partial_{j} \psi_{j n}\right)+\left(\partial_{j} \Phi_{n-1}\right) \psi_{j n}\right)
$$


Proof. For $j=1, \ldots, n-1$

$$
\begin{aligned}
(-1)^{n-1}\left(2 \Phi_{n-1}\left(\partial_{j} \psi_{j n}\right)+\left(\partial_{j} \Phi_{n-1}\right) \psi_{j n}\right) & \\
= & \sum_{\substack{w \in \mathfrak{S}_{n-1} \\
w(n-1)=j}}\left(\partial_{j} \psi_{j n}\right) w\left(\phi_{01} \phi_{12} \cdots \phi_{n-2, n-1}\right) \\
& +\sum_{\substack{w \in \mathfrak{S}_{n-1} \\
w(n-1)=j}}\left(\partial_{j}\left(\phi_{w(n-2) j} \psi_{j n}\right)\right) w\left(\phi_{01} \phi_{12} \cdots \phi_{n-3, n-2}\right) \\
& +\sum_{\substack{w \in \mathfrak{S}_{n-1} \\
w(n-1) \neq j}}\left(\partial_{j}\left(\phi_{w\left(w^{-1}(j)-1\right) j} \psi_{j n}\right)\right) \prod_{\substack{i \neq w(j) \\
1 \leq i \leq n-1}} w\left(\phi_{i-1, i}\right) \\
& +\sum_{\substack{w \in \mathfrak{S}_{n-1} \\
w(n-1) \neq j}}\left(\partial_{j}\left(\phi_{j w\left(w^{-1}(j)+1\right)} \psi_{j n}\right)\right) \prod_{\substack{i \neq w(j) \\
1 \leq i \leq n-1}} w\left(\phi_{i, i+1}\right) .
\end{aligned}
$$

Hence it follows from (2.20) and (2.24) that the right hand side of (5.2) equals

$$
\begin{aligned}
& (-1)^{n}\left(\sum_{j=1}^{n-1} \sum_{\substack{w \in \mathfrak{S}_{n-1} \\
w(n-1)=j}} \partial_{n}\left(\phi_{j n} \prod_{i=1}^{n-1} w\left(\phi_{i-1, i}\right)\right)\right. \\
& \left.-\sum_{w \in \mathfrak{S}_{n-1}} \sum_{k=1}^{n-1} w\left(\partial_{k}\left(\phi_{k-1, k} \psi_{k, n}\right)+\partial_{k-1}\left(\phi_{k-1, k} \psi_{k-1, n}\right)\right) \prod_{\substack{i \neq k \\
1 \leq i \leq n-1}} w\left(\phi_{i-1, i}\right)\right) \\
& =(-1)^{n}\left(\sum_{\substack{w \in \mathfrak{S}_{n} \\
w(n)=n}} \partial_{n}\left(\prod_{i=1}^{n} w\left(\phi_{i-1, i}\right)\right)\right. \\
& \left.+\sum_{w \in \mathfrak{S}_{n-1}} \sum_{k=1}^{n-1} w\left(\partial_{n}\left(\phi_{k-1, n} \phi_{n, k}\right) \prod_{\substack{i \neq k \\
1 \leq i \leq n-1}} \phi_{i-1, i}\right)\right) \\
& =\partial_{n} \Phi_{n} .
\end{aligned}
$$

Thus we have the lemma.

Lemma 5.2. Suppose there exist a symmetric function $g(s, t)$ of $(s, t)$ such that

$$
2 v_{1}\left(\partial_{1} \psi_{12}\right)+\left(\partial_{1} v_{1}\right) \psi_{12}=\partial_{2}\left(2 g_{12}-\left(v_{1}+v_{2}\right) \phi_{12}\right)
$$

by denoting $g_{i j}=g\left(x_{i}, x_{j}\right)$. Put

$$
\begin{aligned}
S_{\{1\}}^{o} & =-2 v_{1}, \\
S_{\{1, \ldots, k\}}^{o} & =2(-1)^{k} \sum_{w \in \mathfrak{S}_{k}} w\left(v_{1} \phi_{12} \phi_{23} \cdots \phi_{k-1, k}\right) \quad \text { for } \quad k \geq 1 .
\end{aligned}
$$


Then

$$
\begin{aligned}
\partial_{k}\left(S_{\{1, \ldots, k\}}^{o}-\right. & \left.(-1)^{k} \sum_{w \in \mathfrak{S}_{k-1}} 4 w\left(g_{1 k} \phi_{12} \phi_{23} \cdots \phi_{k-2, k-1}\right)\right) \\
& =\sum_{j=1}^{k-1}\left(2 S_{\{1, \ldots, k-1\}}^{o}\left(\partial_{j} \psi_{j k}\right)+\left(\partial_{j} S_{\{1, \ldots, k-1\}}^{o}\right) \psi_{j k}\right) .
\end{aligned}
$$

i) If

$$
2 v_{1}\left(\partial_{1} \psi_{12}\right)+\left(\partial_{1} v_{1}\right) \psi_{12}=\partial_{2}\left(2 \lambda v_{1} v_{2}-\left(v_{1}+v_{2}\right) \phi_{12}\right)
$$

with a complex number $\lambda$, then

$$
\begin{cases}S_{\{1\}} & =-2 v_{1}, \\ \partial_{k} S_{\{1, \ldots, k\}} & =\sum_{j=1}^{k-1}\left(2 S_{\{1, \ldots, k-1\}}\left(\partial_{j} \psi_{j k}\right)+\left(\partial_{j} S_{\{1, \ldots, k-1\}}\right) \psi_{j k}\right)\end{cases}
$$

by putting

$$
S_{\{1, \ldots, k\}}=\sum_{I_{1} \amalg \cdots \amalg I_{\nu}=\{1, \ldots, k\}}(-\lambda)^{\nu-1}(\nu-1) ! S_{I_{1}}^{o} \cdots S_{I_{\nu}}^{o} \quad \text { for } \quad k \geq 1 .
$$

ii) If there exist even functions $f(t)$ and $h(t)$ and complex numbers $\lambda, \lambda^{\prime}$ and $\lambda^{\prime \prime}$ such that

$$
\left\{\begin{array}{l}
2 v_{1}\left(\partial_{1} \psi_{12}\right)+\left(\partial_{1} v_{1}\right) \psi_{12}=\partial_{2}\left(2 \lambda^{\prime} f_{1} f_{2}+2 \lambda^{\prime \prime}\left(h_{1}+h_{2}\right)-\left(v_{1}+v_{2}\right) \phi_{12}\right) \\
2 f_{1}\left(\partial_{1} \psi_{12}\right)+\left(\partial_{1} f_{1}\right) \psi_{12}=\partial_{2}\left(-\left(f_{1}+f_{2}\right) \phi_{12}\right) \\
2 h_{1}\left(\partial_{1} \psi_{12}\right)+\left(\partial_{1} h_{1}\right) \psi_{12}=\partial_{2}\left(2 \lambda\left(f_{1}+f_{2}\right)-\left(h_{1}+h_{2}\right) \phi_{12}\right)
\end{array}\right.
$$

the following functions $S_{\{1, \ldots, k\}}$ satisfy (5.7).

$$
\begin{aligned}
S_{\{1, \ldots, k\}}= & S_{\{1, \ldots, k\}}^{o}-\sum_{I_{1} \amalg I_{2}=\{1, \ldots, k\}}\left(\lambda^{\prime} S_{I_{1}}^{\prime} S_{I_{2}}^{\prime}+\lambda^{\prime \prime} S_{I_{1}}^{\prime \prime} D_{I_{2}}+\lambda^{\prime \prime} D_{I_{1}} S_{I_{2}}^{\prime \prime}\right) \\
& +\sum_{I_{1} \amalg I_{2} \amalg I_{3}=\{1, \ldots, k\}} 2 \lambda \lambda^{\prime \prime}\left(S_{I_{1}}^{\prime} D_{I_{2}} D_{I_{3}}+D_{I_{1}} S_{I_{2}}^{\prime} D_{I_{3}}+D_{I_{1}} D_{I_{2}} S_{I_{3}}^{\prime}\right) \\
S_{\{1, \ldots, k\}}^{\prime}= & 2(-1)^{k} \sum_{w \in \mathfrak{S}_{k}} w\left(f_{1} \phi_{12} \phi_{23} \cdots \phi_{k-1, k}\right), \\
S_{\{1, \ldots, k\}}^{\prime \prime}= & 2(-1)^{k} \sum_{w \in \mathfrak{S}_{k}} w\left(h_{1} \phi_{12} \phi_{23} \cdots \phi_{k-1, k}\right), \\
D_{\{1, \ldots, k\}}= & 2(-1)^{k} \sum_{w \in \mathfrak{S}_{k}} w\left(\phi_{12} \phi_{23} \cdots \phi_{k-1, k}\right) .
\end{aligned}
$$

Here we put $f_{j}=f\left(x_{j}\right)$ and $h_{j}=h\left(x_{j}\right)$ for $j \geq 1$ and $S_{\{1\}}^{\prime}=-2 f_{1}, S_{\{1\}}^{\prime \prime}=-2 h_{1}$ and $D_{\{1\}}=-2$.

Proof. Owing to (5.3) and (2.20) and Lemma 5.1, the right hand side of (5.5) equals

$$
2(-1)^{k-1} \sum_{w \in \mathfrak{S}_{k-1}} w\left(2 \sum_{j=1}^{k-1} v_{1} \phi_{12} \cdots \phi_{k-2, k-1}\left(\partial_{j} \psi_{j k}\right)\right.
$$




$$
\begin{aligned}
& \left.+\left(\partial_{1} v_{1}\right) \phi_{12} \cdots \phi_{k-2, k-1} \psi_{1 k}+\sum_{j=1}^{k-1} v_{1}\left(\partial_{j}\left(\phi_{12} \cdots \phi_{k-2, k-1}\right)\right) \psi_{j k}\right) \\
= & 2(-1)^{k} \sum_{w \in \mathfrak{S}_{k-1}} w\left(\partial_{k}\left(v_{k} \phi_{k 1} \phi_{12} \cdots \phi_{k-2, k-1}-2 g_{1 k} \phi_{12} \cdots \phi_{k-2, k-1}\right)\right. \\
& -v_{1} \phi_{12} \cdots \phi_{k-2, k-1}\left(\partial_{1} \psi_{1 k}\right)-2 \sum_{j=2}^{k-1} v_{1} \phi_{12} \cdots \phi_{k-2, k-1}\left(\partial_{j} \psi_{j k}\right) \\
& \left.-\sum_{j=1}^{k-1} v_{1}\left(\partial_{j}\left(\phi_{12} \cdots \phi_{k-2, k-1}\right)\right) \psi_{j k}\right) \\
= & 2(-1)^{k} \sum_{w \in \mathfrak{S}_{k-1}} w\left(\partial _ { k } \left(v_{k} \phi_{k 1} \phi_{12} \cdots \phi_{k-2, k-1}-2 g_{1 k} \phi_{12} \cdots \phi_{k-2, k-1}\right.\right. \\
& \left.\left.+\frac{1}{(k-1) !} \sum_{\substack{w^{\prime} \in \mathfrak{S}_{k} \\
w^{\prime}(1)=1}} w^{\prime}\left(v_{1} \phi_{12} \phi_{23} \cdots \phi_{k-1, k}\right)\right)\right) \\
= & \partial_{k}\left(S_{\{1, \ldots, k\}}^{o}-(-1)^{k} \sum_{w \in \mathfrak{S}_{k-1}} 4 w\left(g_{1 k} \phi_{12} \phi_{23} \cdots \phi_{k-2, k-1}\right)\right) .
\end{aligned}
$$

Hence we have (5.5) and if (5.6) holds, we have

$$
(-1)^{k} \sum_{w \in \mathfrak{S}_{k-1}} 4 w\left(g_{1 k} \phi_{12} \phi_{23} \cdots \phi_{k-2, k-1}\right)=\lambda S_{\{1, \ldots, k-1\}}^{o} S_{\{k\}}^{o}
$$

and therefore the right hand side of the second equation of (5.7) equals

$$
\begin{aligned}
& \sum_{j=1}^{k-1} \sum_{I_{1} \amalg \cdots \amalg I_{\nu}=\{1, \ldots, k-1\}}(-\lambda)^{\nu-1}(\nu-1) !\left(2 S_{I_{1}}^{o} \cdots S_{I_{\nu}}^{o}\left(\partial_{j} \psi_{j k}\right)\right. \\
& \left.\quad+\left(\partial_{j} S_{I_{1}}^{o} \cdots S_{I_{\nu}}^{o}\right) \psi_{j k}\right) \\
& =\sum_{I_{1} \amalg \cdots \amalg I_{\nu}=\{1, \ldots, k-1\}}(-\lambda)^{\nu-1}(\nu-1) ! \sum_{\mu=1}^{\nu} \partial_{k}\left(S_{I_{1}}^{o} \cdots S_{I_{\mu} \cup\{k\}}^{o} \cdots S_{I_{\nu}}^{o}\right. \\
& \left.\quad-\lambda S_{I_{1}}^{o} \cdots S_{I_{\mu}}^{o} \cdots S_{I_{\nu}}^{o} S_{\{k\}}^{o}\right) \\
& =\partial_{k}\left(\sum_{I_{1} \amalg \cdots \amalg I_{\nu}=\{1, \ldots, k\}}(-\lambda)^{\nu-1}(\nu-1) ! S_{I_{1}}^{o} \cdots S_{I_{\nu}}^{o}\right) \\
& =\partial_{k} S_{\{1, \ldots, k\}} \cdot
\end{aligned}
$$

Now suppose (5.9). Then by denoting

$$
\begin{aligned}
S_{\{1, \ldots, k\}}^{1} & =S_{\{1, \ldots, k\}}^{o}, \\
S_{\{1, \ldots, k\}}^{2} & =\sum_{I_{1} \amalg I_{2}=\{1, \ldots, k\}} S_{I_{1}}^{\prime} S_{I_{2}}^{\prime}, \\
S_{\{1, \ldots, k\}}^{3} & =\sum_{I_{1} \amalg I_{2}=\{1, \ldots, k\}}\left(S_{I_{1}}^{\prime \prime} D_{I_{2}}+D_{I_{1}} S_{I_{2}}^{\prime \prime}\right),
\end{aligned}
$$




$$
S_{\{1, \ldots, k\}}^{4}=\sum_{I_{1} \amalg I_{2} \amalg I_{3}=\{1, \ldots, k\}}\left(S_{I_{1}}^{\prime} D_{I_{2}} D_{I_{3}}+D_{I_{1}} S_{I_{2}}^{\prime} D_{I_{3}}+D_{I_{1}} D_{I_{2}} S_{I_{3}}^{\prime}\right)
$$

and

$$
F_{k}^{\nu}=(-1)^{k}\left(\partial_{k} S_{\{1, \ldots, k\}}^{\nu}-\sum_{j=1}^{k-1}\left(2 S_{\{1, \ldots, k-1\}}^{\nu} \partial_{j} \psi_{j k}+\left(\partial_{j} S_{\{1, \ldots, k-1\}}^{\nu}\right) \psi_{j k}\right)\right)
$$

for $\nu=1, \ldots, 4$, it follows from (5.5) that

$$
\begin{aligned}
F_{k}^{1} & =\partial_{k}\left(\lambda^{\prime} S_{\{1, \ldots, k-1\}}^{\prime} S_{\{k\}}^{\prime}+\lambda^{\prime \prime} S_{\{1, \ldots, k-1\}}^{\prime \prime} D_{\{k\}}+\lambda^{\prime \prime} D_{\{1, \ldots, k-1\}} S_{\{k\}}^{\prime \prime}\right), \\
F_{k}^{2} & =\partial_{k}\left(S_{\{1, \ldots, k-1\}}^{\prime} S_{\{k\}}^{\prime}\right), \\
F_{k}^{3} & =\partial_{k}\left(S_{\{1, \ldots, k-1\}}^{\prime \prime} D_{\{k\}}+D_{\{1, \ldots, k-1\}} S_{\{k\}}^{\prime \prime}\right. \\
& \left.+\lambda \sum_{I_{1} \amalg I_{2}=\{1, \ldots, k-1\}}\left(\left(S_{I_{1}}^{\prime} D_{\{k\}}+D_{I_{1}} S_{\{k\}}^{\prime}\right) D_{I_{2}}+D_{I_{1}}\left(S_{I_{2}}^{\prime} D_{\{k\}}+D_{I_{2}} S_{\{k\}}^{\prime}\right)\right)\right), \\
F_{k}^{4} & =\partial_{k}\left(\sum_{I_{1} \amalg I_{2}=\{1, \ldots, k-1\}}\left(S_{I_{1}}^{\prime} D_{I_{2}} D_{\{k\}}+D_{I_{1}} S_{I_{2}}^{\prime} D_{\{k\}}+D_{I_{1}} D_{I_{2}} S_{\{k\}}^{\prime}\right) .\right.
\end{aligned}
$$

Since $\partial_{k}\left(\left(S_{I_{1}}^{\prime} D_{I_{2}}+D_{I_{1}} S_{I_{2}}^{\prime}\right) D_{\{k\}}\right)=0$ in the above, we have

$$
F_{k}^{1}-\lambda^{\prime} F_{k}^{2}-\lambda^{\prime \prime} F_{k}^{3}+2 \lambda \lambda^{\prime \prime} F_{k}^{4}=0,
$$

which implies (5.7). Thus we have completed the proof of the lemma.

Definition 5.3. For given even functions $f$ and $g$ of $t$, we define

$$
\begin{aligned}
& \Phi_{\{1, \ldots, k\}}(f, g)=\sum_{w \in W\left(B_{k}\right)} w\left(f\left(x_{1}\right) g\left(x_{1}-x_{2}\right) g\left(x_{2}-x_{3}\right) \cdots g\left(x_{k-1}-x_{k}\right)\right), \\
& \Theta_{\{1, \ldots, k\}}(f, g)=\sum_{I_{1} \amalg \cdots \amalg I_{\nu}=\{1, \ldots, k\}}(-1)^{\nu-1}(\nu-1) ! \Phi_{I_{1}}(f, g) \cdots \Phi_{I_{\nu}}(f, g)
\end{aligned}
$$

for $k \geq 1$. Here we note that $\Theta_{\{1\}}(f, g)=\Phi_{\{1\}}(f, g)=2 f\left(x_{1}\right)$ and $\Theta_{\varnothing}(f, g)=$ $\Phi_{\varnothing}(f, g)=0$.

Proposition 5.4. Suppose

$$
u(t)=w(t)=C_{5} \wp(t), \quad v(t)=\sum_{j=1}^{4} C_{j} \wp\left(t+\omega_{j}\right)-\frac{C_{0}}{2}
$$

with $C_{0}, \ldots, C_{5} \in \mathbb{C}$. Then (4.4) holds by putting

$$
T_{\{1, \ldots, k\}}=\left(-C_{5}\right)^{k-1}\left(\frac{C_{0}}{2} \Theta_{\{1, \ldots, k\}}(1, \wp(t))-\sum_{j=1}^{4} C_{j} \Theta_{\{1, \ldots, k\}}\left(\wp\left(t+\omega_{j}\right), \wp(t)\right)\right) .
$$

Proof. Suppose $C_{5}=1$. If $v(t)=\wp\left(t+\omega_{\nu}\right)$ with $\nu=1, \ldots, 4$, the assumption (5.3) in Lemma 5.2 holds with $g_{12}=v_{1} v_{2}$. In fact (2.27) means (5.3) if $v(t)=\wp(t)$ and then the coordinate transformation $x_{j} \mapsto x_{j}+\omega_{\nu}$ for $j=1, \ldots, n$ implies the case when $v(t)=\wp\left(t+\omega_{\nu}\right)$. If $v$ is constant, then (5.3) is also valid with $g_{12}=v_{1} v_{2}$. Hence the proposition follows from Lemma 5.2 and Remark 4.3.

Remark 5.5. Since we may put $g_{12}=0$ in the above proof when $v$ is constant, we may replace $\Theta_{\{1, \ldots, k\}}(1, \wp(t))$ by $\Phi_{\{1, \ldots, k\}}(1, \wp(t))$ in Proposition 5.4. 


\section{Degenerate solutions of the functional differential equation.}

We give trigonometric and rational solutions of (4.4):

Proposition 6.1. For complex numbers $\lambda, C_{0}, \ldots, C_{5}$ with $\lambda \neq 0$, put

$$
\begin{aligned}
u(t)= & w(t)=C_{5} \sinh ^{-2} \lambda t, \\
v(t)= & C_{1} \sinh ^{-2} \lambda t+C_{2} \cosh ^{-2} \lambda t+C_{3} \sinh ^{2} \lambda t+\frac{C_{4}}{4} \sinh ^{2} 2 \lambda t-\frac{C_{0}}{2}, \\
g(s, t)= & C_{5}\left(C_{1} \sinh ^{-2} \lambda s \cdot \sinh ^{-2} \lambda t-C_{2} \cosh ^{-2} \lambda s \cdot \cosh ^{-2} \lambda t\right. \\
& \left.+C_{4}\left(\sinh ^{2} \lambda s+\sinh ^{2} \lambda t+2 \sinh ^{2} \lambda s \cdot \sinh ^{2} \lambda t\right)\right) .
\end{aligned}
$$

Then (5.3) holds. Moreover we have (4.4) with

$$
\begin{aligned}
T_{I}= & \left(-C_{5}\right)^{\# I-1}\left(\frac{C_{0}}{2} T_{I}^{o}(1)-C_{1} T_{I}^{o}\left(\sinh ^{-2} \lambda t\right)-C_{2} T_{I}^{o}\left(\cosh ^{-2} \lambda t\right)\right. \\
& \left.-C_{3} T_{I}^{o}\left(\sinh ^{2} \lambda t\right)-C_{4} T_{I}^{o}\left(\frac{1}{4} \sinh ^{2} 2 \lambda t\right)\right)
\end{aligned}
$$

by putting

$$
\begin{aligned}
T_{I}^{o}(1) & =\Phi_{I}(1, \rho), \\
T_{I}^{o}\left(\sinh ^{-2} \lambda t\right)= & \Theta_{I}\left(\sinh ^{-2} \lambda t, \rho\right), \\
T_{I}^{o}\left(\cosh ^{-2} \lambda t\right)= & -\Theta_{I}\left(-\cosh ^{-2} \lambda t, \rho\right), \\
T_{I}^{o}\left(\sinh ^{2} \lambda t\right)= & \Phi_{I}\left(\sinh ^{2} \lambda t, \rho\right), \\
T_{I}^{o}\left(\frac{1}{4} \sinh ^{2} 2 \lambda t\right)= & \Phi_{I}\left(\frac{1}{4} \sinh ^{2} 2 \lambda t, \rho\right)-\sum_{I_{1} \amalg I_{2}=I}\left(2 \Phi_{I_{1}}\left(\sinh ^{2} \lambda t, \rho\right) \cdot \Phi_{I_{2}}\left(\sinh ^{2} \lambda t, \rho\right)\right. \\
& \left.+\Phi_{I_{1}}\left(\sinh ^{2} \lambda t, \rho\right) \cdot \Phi_{I_{2}}(1, \rho)+\Phi_{I_{1}}(1, \rho) \cdot \Phi_{I_{2}}\left(\sinh ^{2} \lambda t, \rho\right)\right),
\end{aligned}
$$

where $I \subset\{1, \ldots, n\}, \rho=\sinh ^{-2} \lambda t$ and the last sum runs over different partitions.

Proof. We can prove (5.3) by direct calculations but here we do it in a different way. First note that we may assume that $C_{5}$ and one of the numbers $C_{1}, \ldots, C_{4}$ equal 1 and that the other 4 numbers are 0 . Also by a simple change of coordinates we may assume $\lambda=1$.

Now put $u(t)=\wp(t)-e_{1}$. Then if $v(t)=\wp\left(t+\omega_{j}\right)-e_{1}$ we have (5.3) with $g_{12}=v_{1} v_{2}$ for $j=1, \ldots, 4$. If $e_{1}=e_{2}=\frac{1}{3}$ and $e_{3}=-\frac{2}{3}$, then $\wp(t)-e_{1}=\sinh ^{-2} t$ and

$$
\begin{aligned}
\wp\left(t+\omega_{3}\right)-e_{1} & =e_{3}-e_{1}+\frac{\left(e_{3}-e_{1}\right)\left(e_{3}-e_{2}\right)}{\wp(t)-e_{3}} \\
& =-1+\frac{1}{\sinh ^{-2} t+1}=-\cosh ^{-2} t .
\end{aligned}
$$

Hence if $u(t)=\sinh ^{-2} t$ and $v(t)=\sinh ^{-2} t$ or $-\cosh ^{-2} t$, we have (5.3) with $g_{12}=$ $v_{1} v_{2}$.

Put $e_{1}=\frac{1}{3}, e_{2}=\frac{1}{3}-\varepsilon, e_{3}=-\frac{2}{3}+\varepsilon$ with $0<|\varepsilon|<<1$. Then it follows from (2.8) that

$$
\wp\left(t+\omega_{1}\right)-e_{1}=\frac{\left(e_{1}-e_{2}\right)\left(e_{1}-e_{3}\right)}{\wp(t)-e_{1}}=\varepsilon \sinh ^{2} t+o(\varepsilon) .
$$


Hence putting $v(t)=\wp\left(t+\omega_{1}\right)-e_{1}$, the coefficients of $\varepsilon$ in (5.3) proves (5.3) for $(u, v)=\left(\sinh ^{-2} t, \sinh ^{2} t\right)$ with $g(s, t)=0$.

Next suppose $v(t)=\left(\wp\left(t+\omega_{1}\right)-e_{1}\right)+\left(\wp\left(t+\omega_{2}\right)-e_{1}\right)+\left(e_{1}-e_{2}\right)$. Then

$$
\begin{aligned}
v(t) & =\varepsilon\left(\frac{e_{1}-e_{3}}{\wp(t)-e_{1}}-\frac{e_{2}-e_{3}}{\wp(t)-e_{2}}\right)=\varepsilon^{2} \frac{\wp(t)-e_{3}}{\left(\wp(t)-e_{1}\right)\left(\wp(t)-e_{2}\right)} \\
& =\varepsilon^{2} \frac{\sinh ^{-2} t+1}{\sinh ^{-4} t}+o\left(\varepsilon^{2}\right)=\frac{\varepsilon^{2}}{4} \sinh ^{2} 2 t+o\left(\varepsilon^{2}\right)
\end{aligned}
$$

and (5.3) holds with

$$
\begin{aligned}
g(s, t) & =\left(\wp\left(s+\omega_{1}\right)-e_{1}\right)\left(\wp\left(t+\omega_{1}\right)-e_{1}\right)+\left(\wp\left(s+\omega_{2}\right)-e_{1}\right)\left(\wp\left(t+\omega_{2}\right)-e_{1}\right) \\
& =\left(\varepsilon \sinh ^{2} s\right)\left(\varepsilon \sinh ^{2} t\right)+\left(-\varepsilon \sinh ^{2} s-\varepsilon\right)\left(-\varepsilon \sinh ^{2} t-\varepsilon\right)+o\left(\varepsilon^{2}\right) \\
& =\varepsilon^{2}\left(1+\sinh ^{2} s+\sinh ^{2} t+2 \sinh ^{2} s \cdot \sinh ^{2} t\right)+o\left(\varepsilon^{2}\right) .
\end{aligned}
$$

Hence we have (5.3) if

$$
\left\{\begin{array}{l}
g(s, t)=\sinh ^{2} s+\sinh ^{2} t+2 \sinh ^{2} s \cdot \sinh ^{2} t \\
(u, v)=\left(\sinh ^{-2} t, \frac{1}{4} \sinh ^{2} 2 t\right)
\end{array}\right.
$$

The remaining part of the proposition is clear from Lemma 5.2 and Remark 4.3. We can also get it from Proposition 5.4 by considering the limit as above.

Remark 6.2. Since

$$
\begin{aligned}
\sinh ^{2} \lambda s+ & \sinh ^{2} \lambda t+2 \sinh ^{2} \lambda s \cdot \sinh ^{2} \lambda t \\
& =\sinh ^{2} \lambda s \cdot \sinh ^{2} \lambda t+\cosh ^{2} \lambda s \cdot \cosh ^{2} \lambda t-1,
\end{aligned}
$$

we may put

$$
\begin{aligned}
& T_{I}^{o}\left(\frac{1}{4} \sinh ^{2} 2 \lambda t\right)=\Phi_{I}\left(\frac{1}{4} \sinh ^{2} 2 \lambda t, \rho\right) \\
& \quad-\sum_{I_{1} \amalg I_{2}=I}\left(\Phi_{I_{1}}\left(\sinh ^{2} \lambda t, \rho\right) \cdot \Phi_{I_{2}}\left(\sinh ^{2} \lambda t, \rho\right)+\Phi_{I_{1}}\left(\cosh ^{2} \lambda t, \rho\right) \cdot \Phi_{I_{2}}\left(\cosh ^{2} \lambda t, \rho\right)\right)
\end{aligned}
$$

in Proposition 6.1.

Proposition 6.3. For complex numbers $C_{0}, \ldots, C_{5}$, put

$$
\begin{aligned}
u(t) & =w(t)=C_{5} t^{-2}, \\
v(t) & =C_{1} t^{-2}+C_{2} t^{2}+C_{3} t^{4}+C_{4} t^{6}-\frac{C_{0}}{2}, \\
g(s, t) & =C_{5}\left(C_{1} s^{-2} t^{-2}+C_{3}\left(s^{2}+t^{2}\right)+C_{4}\left(s^{4}+t^{4}+3 s^{2} t^{2}\right)\right) .
\end{aligned}
$$

Then (5.3) holds. Moreover we have (4.4) with

$$
T_{I}=\left(-C_{5}\right)^{\# I-1}\left(\frac{C_{0}}{2} T_{I}^{o}(1)-C_{1} T_{I}^{o}\left(t^{-2}\right)-C_{2} T_{I}^{o}\left(t^{2}\right)-C_{3} T_{I}^{o}\left(t^{4}\right)-C_{4} T_{I}^{o}\left(t^{6}\right)\right)
$$

by putting

$$
\begin{aligned}
T_{I}^{o}(1) & =\Phi_{I}(1, \rho), \\
T_{I}^{o}\left(t^{-2}\right) & =\Theta_{I}\left(t^{-2}, \rho\right), \\
T_{I}^{o}\left(t^{2}\right) & =\Phi_{I}\left(t^{2}, \rho\right), \\
T_{I}^{o}\left(t^{4}\right) & =\Phi_{I}\left(t^{4}, \rho\right)-\sum_{I_{1} \amalg I_{2}=\{1, \ldots, k\}}\left(\Phi_{I_{1}}\left(t^{2}, \rho\right) \cdot \Phi_{I_{2}}(1, \rho)+\Phi_{I_{1}}(1, \rho) \cdot \Phi_{I_{2}}\left(t^{2}, \rho\right)\right),
\end{aligned}
$$




$$
\begin{aligned}
T_{I}^{o}\left(t^{6}\right)= & \Phi_{I}\left(t^{6}, \rho\right)-\sum_{I_{1} \amalg I_{2}=\{1, \ldots, k\}}\left(3 \Phi_{I_{1}}\left(t^{2}, \rho\right) \cdot \Phi_{I_{2}}\left(t^{2}, \rho\right)\right. \\
& \left.+\Phi_{I_{1}}\left(t^{4}, \rho\right) \cdot \Phi_{I_{2}}(1, \rho)+\Phi_{I_{1}}(1, \rho) \cdot \Phi_{I_{2}}\left(t^{4}, \rho\right)\right) \\
+ & \sum_{\substack{I_{1} \amalg I_{2} \amalg I_{3}=\{1, \ldots, k\}\\
}} 6\left(\Phi_{I_{1}}\left(t^{2}, \rho\right) \cdot \Phi_{I_{2}}(1, \rho) \cdot \Phi_{I_{3}}(1, \rho)\right. \\
& \left.+\Phi_{I_{1}}(1, \rho) \cdot \Phi_{I_{2}}\left(t^{2}, \rho\right) \cdot \Phi_{I_{3}}(1, \rho)+\Phi_{I_{1}}(1, \rho) \cdot \Phi_{I_{2}}(1, \rho) \cdot \Phi_{I_{3}}\left(t^{2}, \rho\right)\right),
\end{aligned}
$$

where $I \subset\{1, \ldots, n\}, \rho=t^{-2}$ and the sums run over different partitions.

Proof. Note that the proof proceeds in the same way as in the proof of Proposition 6.1. Put $u(t)=\lambda^{2} \sinh ^{-2} \lambda t$. Then for

$$
\left\{\begin{array}{l}
v(t)=\lambda^{2} \sinh ^{-2} \lambda t \\
g(s, t)=\lambda^{4} \sinh ^{-2} \lambda s \cdot \sinh ^{-2} \lambda t
\end{array}\right.
$$

or

$$
\left\{\begin{array}{l}
v(t)=\lambda^{-2} \sinh ^{2} \lambda t \\
g(s, t)=0
\end{array}\right.
$$

or

$$
\left\{\begin{array}{l}
v(t)=\lambda^{-4}\left(\frac{1}{4} \sinh ^{2} 2 \lambda t-\sinh ^{2} \lambda t\right), \\
g(s, t)=\lambda^{-2}\left(\sinh ^{2} \lambda s+\sinh ^{2} \lambda t+2 \sinh ^{2} \lambda s \cdot \sinh ^{2} \lambda t\right)
\end{array}\right.
$$

or

$$
\left\{\begin{aligned}
v(t)= & \lambda^{-6}\left(1-2 \sinh ^{2} \lambda t+\frac{1}{4} \sinh ^{2} 2 \lambda t-\cosh ^{-2} \lambda t\right), \\
g(s, t)= & \lambda^{-4}\left(\cosh ^{2} \lambda s \cdot \cosh ^{2} \lambda t+\sinh ^{2} \lambda s \cdot \sinh ^{2} \lambda t\right. \\
& \left.+\cosh ^{-2} \lambda s \cdot \cosh ^{-2} \lambda t-2\right),
\end{aligned}\right.
$$

we have (5.3). By the analytic continuation of these $u(t), v(t)$ and $g(s, t)$ to $\lambda=0$, we have (5.3) for $u(t)=t^{-2}$ and $v(t)=t^{-2}$ or $t^{2}$ or $t^{4}$ or $t^{6}$ with $g(s, t)=s^{-2} t^{-2}$ or 0 or $s^{2}+t^{2}$ or $s^{4}+t^{4}+3 s^{2} t^{2}$, respectively. In fact, for example, we have

$$
\begin{aligned}
& \lambda^{-6}\left(1-2 \sinh ^{2} \lambda t+\frac{1}{4} \sinh ^{2} 2 \lambda t-\cosh ^{-2} \lambda t\right) \\
&= \lambda^{-6}\left(1-\sinh ^{2} \lambda t+\sinh ^{4} \lambda t-\left(1+\sinh ^{2} \lambda t\right)^{-1}\right) \\
&= \lambda^{-6} \sinh ^{6} \lambda t+o(\lambda)=t^{6}+o(\lambda), \\
& \lambda^{-4}\left(\cosh ^{2} \lambda s \cdot \cosh ^{2} \lambda t+\sinh ^{2} \lambda s \cdot \sinh ^{2} \lambda t+\cosh ^{-2} \lambda s \cdot \cosh ^{-2} \lambda t-2\right) \\
&=\lambda^{-4}\left(\left(1+\sinh ^{2} \lambda s\right)\left(1+\sinh ^{2} \lambda t\right)+\left(1+\sinh ^{2} \lambda s\right)^{-1}\left(1+\sinh ^{2} \lambda t\right)^{-1}\right. \\
&\left.\quad+\sinh ^{2} \lambda s \cdot \sinh ^{2} \lambda t-2\right) \\
&=\lambda^{-4}\left(\left(\sinh ^{2} \lambda s+\sinh ^{2} \lambda t\right)^{2}+\sinh ^{2} \lambda s \cdot \sinh ^{2} \lambda t\right)+o(\lambda) \\
&=s^{4}+t^{4}+3 s^{2} t^{2}+o(\lambda) .
\end{aligned}
$$

The remaining part of the proposition is clear from Lemma 5.2 and Remark 4.3. We can also get it from Proposition 6.1 by taking the limit at $\lambda=0$.

7. Integrals of type $B_{n}$ and $D_{n}$.

The argument in the preceding sections gives the integrals when $W$ is of type $B_{n}$ or $D_{n}$. 
Definition 7.1. For given even function $u(t)$ and symmetric functions $T_{\{1, \ldots, k\}}$ of $\left(x_{1}, \ldots, x_{k}\right)$ for $k=1, \ldots, n$, define $W\left(B_{n}\right)$-invariant differential operator

$$
P(u, T)=\sum_{k=0}^{n} \frac{1}{k !(n-k) !} \sum_{w \in \mathfrak{S}_{n}} w\left(q_{\{1, \ldots, k\}} \Delta_{\{k+1, \ldots, n\}}^{2}\right)
$$

by

$$
\begin{aligned}
\Delta_{\{1, \ldots, k\}}= & \sum_{0 \leq j \leq\left[\frac{k}{2}\right]} \frac{1}{2^{k} j !(k-2 j) !} \sum_{w \in W\left(B_{k}\right)} \varepsilon(w) w\left(u\left(x_{1}-x_{2}\right) u\left(x_{3}-x_{4}\right) \cdots\right. \\
& \left.\cdot u\left(x_{2 j-1}-x_{2 j}\right) \partial_{2 j+1} \partial_{2 j+2} \cdots \partial_{k}\right) \\
q_{\{1, \ldots, k\}}= & \sum_{I_{1} \amalg \cdots \amalg I_{\nu}=\{1, \ldots, k\}} T_{I_{1}} \cdots T_{I_{\nu}}
\end{aligned}
$$

where

$$
\begin{aligned}
q_{\varnothing} & =1, q_{\{1\}}=T_{\{1\}}, \quad q_{\{12\}}=T_{\{1\}} T_{\{2\}}+T_{\{1,2\}}, \ldots \\
T_{w(\{1, \ldots, k\})} & =w\left(T_{\{1, \ldots, k\}}\right), \quad \Delta_{w(\{1, \ldots, k\})}=w\left(\Delta_{\{1, \ldots, k\}}\right) \text { for } w \in \mathfrak{S}_{n} .
\end{aligned}
$$

Replacing $\partial_{i}$ by $\xi_{i}$ for $i=1, \ldots, n$ in the definition of $\Delta_{\{1, \ldots, k\}}$ and $P(u, T)$, we define functions $\bar{\Delta}_{\{1, \ldots, k\}}$ and $\bar{P}(u, T)$ of $(x, \xi)$, respectively.

Theorem 7.2 (Elliptic Potentials: Generic cases of Type $B_{n}$ ). Put

$$
\left\{\begin{array}{l}
u(t)=C_{5} \wp(t), \\
v(t)=\sum_{j=1}^{4} C_{j} \wp\left(t+\omega_{j}\right)-\frac{C_{0}}{2}
\end{array}\right.
$$

and define $P_{n}\left(C_{0}\right)=P(u, T)$ and $\bar{P}_{n}\left(C_{0}\right)=\bar{P}(u, T)$ by

$$
\begin{aligned}
T_{\{1, \ldots, k\}} & =\left(-C_{5}\right)^{k-1}\left(\frac{C_{0}}{2} T_{\{1, \ldots, k\}}^{o}(1)-\sum_{j=1}^{4} C_{j} T_{\{1, \ldots, k\}}^{o}\left(\wp\left(t+\omega_{j}\right)\right)\right), \\
T_{\{1, \ldots, k\}}^{o}(\psi) & =\sum_{I_{1} \amalg \cdots \amalg I_{\nu}=\{1, \ldots, k\}}(-1)^{\nu-1}(\nu-1) ! S_{I_{1}}(\psi) \cdots S_{I_{\nu}}(\psi), \\
S_{\{1, \ldots, k\}}(\psi) & =\sum_{w \in W\left(B_{k}\right)} w\left(\psi\left(x_{1}\right) \wp\left(x_{1}-x_{2}\right) \wp\left(x_{2}-x_{3}\right) \cdots \wp\left(x_{k-1}-x_{k}\right)\right) .
\end{aligned}
$$

Then

$$
\left[P_{n}(C), P_{n}\left(C^{\prime}\right)\right]=\left\{\bar{P}_{n}(C), \bar{P}_{n}\left(C^{\prime}\right)\right\}=0
$$

for $C, C^{\prime} \in \mathbb{C}$.

Let $P_{j}$ be the coefficient of $C_{0}^{n-j}$ in $P_{n}\left(C_{0}\right)$. Then $P_{1}, \ldots, P_{n}$ are the required commuting differential operators (1.5) for the Schrödinger operator

$$
P=-\frac{1}{2} \sum_{j=1}^{n} \frac{\partial^{2}}{\partial x_{j}^{2}}+\sum_{1 \leq i<j \leq n}\left(u\left(x_{i}-x_{j}\right)+u\left(x_{i}+x_{j}\right)\right)+\sum_{k=1}^{n} v\left(x_{k}\right)
$$

in the case when $W$ is of type $B_{n}$. 
By using $\bar{P}_{n}\left(C_{0}\right)$ in place of $P_{n}\left(C_{0}\right)$, we have integrals $\bar{P}_{j}$ of the Hamiltonian

$$
\bar{P}=-\frac{1}{2} \sum_{j=1}^{n} \xi_{j}^{2}+\sum_{1 \leq i<j \leq n}\left(u\left(x_{i}-x_{j}\right)+u\left(x_{i}+x_{j}\right)\right)+\sum_{k=1}^{n} v\left(x_{k}\right),
$$

where $\bar{P}_{1}, \ldots, \bar{P}_{n}$ are functionally independent and satisfy $\left\{\bar{P}_{i}, \bar{P}_{j}\right\}=0$.

Proof. Theorem 4.2 and Proposition 5.4 imply

$$
\left[P_{n}\left(C_{0}\right), P\right]=0 .
$$

Fix $C, C^{\prime} \in \mathbb{C}$ and put $Q=\left[P_{n}(C), P_{n}\left(C^{\prime}\right)\right]$. Then we have $[Q, P]=0$ and $Q^{-}=$ ${ }^{-}{ }^{t} Q=Q$ and therefore we have $Q=0$ as in the proof of Theorem 3.2.

Since $q_{\{1, \ldots, k\}}$ is a monic polynomial of $C_{0}$ with degree $k$, it is clear that $P_{j}$ for $j=1, \ldots, n$ satisfy (1.5). The remaining part of the theorem is also clear.

Theorem 7.3 (Type $D_{n}$ ). Suppose $W$ is of type $D_{n}$. Then by putting $C_{1}=$ $C_{2}=C_{3}=C_{4}=0$, the operators $P_{1}, \ldots, P_{n-1}$ in Theorem 7.2 and $P_{n}=\Delta_{\{1, \ldots, n\}}$ are the required commuting differential operators (1.6) for the Schrödinger operator

$$
P=-\frac{1}{2} \sum_{j=1}^{n} \frac{\partial^{2}}{\partial x_{j}^{2}}+\sum_{1 \leq i<j \leq n}\left(u\left(x_{i}-x_{j}\right)+u\left(x_{i}+x_{j}\right)\right)
$$

with the function $u(t)$ given by (7.1). Here the periods of $\wp(t)$ are allowed to be infinity.

Proof. Theorem 7.2 and Proposition 3.1 prove $\left[P_{j}, P\right]=0$ for $j=1, \ldots, n$. Then the commutators $Q_{j}=\left[P_{j}, P_{n}\right]$ satisfy $Q_{j}^{-}=-{ }^{t} Q_{j}=(-1)^{n} Q_{j}$ and $\left[Q_{j}, P\right]=0$ and hence $Q_{j}=0$ as in the proof of Theorem 3.2.

Remark 7.4. i) In Theorem 7.2 we have $P_{n}=P_{n}(0)$ and

$$
\begin{aligned}
P_{n-k}= & \sum_{i=k}^{n} \sum_{j=i}^{n} \frac{1}{i !(j-i) !(n-j) !} \sum_{w \in \mathfrak{S}_{n}} \sum_{I_{1} \amalg \cdots \amalg I_{k}=\{1, \ldots, i\}} \\
& w\left(\left(-C_{5}\right)^{i-k} 2^{-k} T_{I_{1}}^{o}(1) \cdots T_{I_{k}}^{o}(1) q_{\{i+1, \ldots, j\}} \Delta_{\{j+1, \ldots, n\}}^{2}\right)
\end{aligned}
$$

for $k=1, \ldots, n-1$, where $q_{\{i+1, \ldots, j\}}$ are defined by putting $C_{0}=0$.

ii) Because of the uniqueness of $\mathbb{C}\left[P_{1}, \ldots, P_{n}\right]$ in terms of $(u, v)$ (cf. [OS, Theorem 6.5]), the existence of the commuting differential operators $P_{1}, \ldots, P_{n}$ for (1.10) which satisfy (1.5) is guaranteed by the analytic continuation of the parameters $g_{2}$ and $g_{3}$ of $\wp(t)$ even if $\omega_{1}$ or $\omega_{2}$ is infinite. We have explicitly given the analytic continuation. In fact Theorem 4.2, Proposition 6.1, Proposition 6.3 and the proof of Theorem 7.2 imply the following theorem.

THEOREM 7.5 (Degenerate cases of Type $B_{n}$ ). Suppose

i) Trigonometric Potentials:

$$
\left\{\begin{array}{l}
u(t)=C_{5} \sinh ^{-2} \lambda t, \\
v(t)=C_{1} \sinh ^{-2} \lambda t+C_{2} \cosh ^{-2} \lambda t+C_{3} \sinh ^{2} \lambda t+\frac{C_{4}}{4} \sinh ^{2} 2 \lambda t-\frac{C_{0}}{2}
\end{array}\right.
$$

with a non-zero complex number $\lambda$ or

ii) Rational Potentials:

$$
\left\{\begin{array}{l}
u(t)=C_{5} t^{-2} \\
v(t)=C_{1} t^{-2}+C_{2} t^{2}+C_{3} t^{4}+C_{4} t^{6}-\frac{C_{0}}{2} .
\end{array}\right.
$$


Then for the function $T_{\{1, \ldots, k\}}$ defined in Proposition 6.1 or Proposition 6.3, we have the same statements as in Theorem 7.2.

\section{REFERENCES}

[Be] F. A. Berezin, Laplace operators on semisimple Lie groups, Proc. Mosc. Math. Soc., 6 (1971), pp. 371-463. (Russian)

[BBy] H. W. Braden AND J. G. B. ByatT-Smith, On a functional differential equation of determinantal type, math.CA/9804082, preprint.

[BBu] H. W. BRADEN AND V. M. BUCHSTABER, Integrable systems with pairwise interactions and functional equations, Reviews in Mathematics and Mathematical Physics, 10(2) (1997), pp. 121-166.

[BP] V. M. Buchstaber And A. M. Perelomov, On the functional equation related to the quantum three-body problem, Contemporary Mathematical Physics, Amer. Math. Soc. Transl. Ser. 2, 175 (1996), pp. 15-34.

[Ca] F. CALOgero, Solution of the one dimensional $n$-body problem with quadratic and/or inverse quadratic pair potentials, J. Math. Phys., 12 (1971), pp. 419-436.

[Ch] I. CHEREDNIK, Elliptic quantum many-body problem and double affine Knizhnik-Zamolodohikov equation, Commun. Math. Phys., 169 (1995), pp. 441-461.

[De] A. DEBIARD, Systèm différentiel hypergeómétrique et parties radiales des espaces symétriques de type $B C_{p}$, Springer Lecture Notes in Math., 1296 (1988), pp. 42-124.

[Et] P. I. ETINgof, Quantum integrable systems and representations of Lie algebras, J. Math. Phys., 36 (1995), pp. 2636-2651.

[He1] G. J. Heckman, Root system and hypergeometric functions II, Comp. Math., 64 (1987), pp. 353-373.

[He2] ——, An elementary approach to the hypergeometric shift operators of Opdam, Invent. Math., 103 (1991), pp. 341-350.

[HO] G. J. Heckman And E. M. Opdam, Root system and hypergeometric functions I, Comp. Math., 64 (1987), pp. 329-352.

[In] V. I. INOZEMTSEV, Lax representation with spectral parameter on a torus for Integrable particle systems,, Lett. Math. Phys., 17 (1989), pp. 11-17.

[Oc] H. OCHIAI, Commuting differential operators of rank two, Indag. Math. (N.S.), 7 (1996), pp. 243-255.

[OO] H. OCHIAI AND T. OshIMA, Commuting differential operators with $B_{2}$ symmetry, UTMS 94-65, Dept. of Mathematical Sciences, Univ. of Tokyo, 1994, pp. 1-31, preprint.

[OOS] H. Ochiai, T. Oshima, and H. Sekiguchi, Commuting families of symmetric differential operators, Proc. Japan Acad., 70A (1994), pp. 62-66.

[OP1] M. A. Olshanetsky and M. A. Perelomov, Classical integrable finite dimensional systems related to Lie algebras, Phys. Rep., 71 (1981), pp. 313-400.

[OP2] — Quantum integrable systems related to Lie algebras, Phys. Rep., 94 (1983), pp. 313404.

[Op1] E. M. OPDAM, Root system and hypergeometric functions III, Comp. Math., 67 (1988), pp. 21-49.

[Op2] - Root system and hypergeometric functions IV, Comp. Math., 67 (1988), pp. 191-209.

[Os] T. Oshima, Completely integrable systems with a symmetry in coordinates, UTMS 94-6, Dept. of Mathematical Sciences, Univ. of Tokyo, 1994, pp. 1-22, preprint.

[OS] T. Oshima And H. Sekiguchi, Commuting families of differential operators invariant under the action of a Weyl group, J. Math. Sci. Lniv. Tokyo, 2 (1995), pp. 1-75.

[Sj] J. SEKIGUCHI, Zonal spherical functions on some symmetric spaces, Rubl. RIMS Kyoto Univ., 12 Suppl. (1977), pp. 455-459.

[Su] B. SutherLAND, Exact results for a quantum many-body problem in one dimension, Phys. Rev., A5 (1972), pp. 1372-1376.

[WW] E. T. Whittaker and G. N. Watson, A Course of Modern Analysis, Fourth Edition, Cambridge University Press, 1927. 
T. OSHIMA 